\title{
MEMORY BUT NO SUPPRESSION IN LOW-DIMENSIONAL SYMMETRIC IDIOTYPIC NETWORKS
}

\author{
- Rob J. De Boer and Pauline Hogeweg \\ Bioinformatics Group, \\ University of Utrecht, \\ Padualaan 8, 3584 CH Utrecht, \\ The Netherlands
}

We present a new symmetric model of the idiotypic immune network. The model specifies clones of B-lymphocytes and incorporates: (1) influx and decay of cells; (2) symmetric stimulatory and inhibitory idiotypic interactions; (3) an explicit affinity parameter (matrix); (4) external (i.e. nonidiotypic) antigens. Suppression is the dominant interaction, i.e. strong idiotypic interactions are always suppressive. This precludes reciprocal stimulation of large clones and thus infinite proliferation. Idiotypic interactions first evoke proliferation, this enlarges the clones, and may in turn evoke suppression. We investigate the effect of idiotypic interactions on normal proliferative immune responses to antigens (e.g. viruses).

A 2-D, i.e. two clone, network has a maximum of three stable equilibria: the virgin state and two asymmetric immune states. The immune states only exist if the affinity of the idiotypic interaction is high enough. Stimulation with antigen leads to a switch from the virgin state to the corresponding immune state. The network therefore remembers antigens, i.e. it accounts for immunity/memory by switching beteen multiple stable states. 3-D systems have, depending on the affinities, 9 qualitatively different states. Most of these also account for memory by state switching.

Our idiotypic network however fails to account for the control of proliferation, e.g. suppression of excessive proliferation. In symmetric networks, the proliferating clones suppress their anti-idiotypic suppressors long before the latter can suppress the former. The absence of proliferation control violates the general assumption that idiotypic interactions play an important role in immune regulation. We therefore test the robustness of these results by abandoning our assumption that proliferation occurs before suppression. We thus define an "escape from suppression" model, i.e. in the "virgin" state idiotypic interactions are now suppressive. This system erratically accounts for memory and never for suppression. We conclude that our "absence of suppression from idiotypic interactions" does not hinge upon our "proliferation before suppression" assumption.

1. Introduction. The immune system consists of a large number $\left(>10^{7}\right)$ of different clones of lymphocytes. The lymphocytes comprising such a clone all share identical antigen receptors. The variable (V) regions of these receptors are generated by random processes such as somatic recombination and somatic mutation (Early et al.,'1980; Berek et al., 1985). The primary repertoire can therefore be visualized as a large (random) array of receptor molecules. Out of the total array of (random) antigen receptors, an antigen "selects" those structures (those paratopes) that provide a mirror image of the antigenic structure (the epitope, Jerne, 1974). This is clonal selection theory (Burnet, 
1959). Only the clones with a receptor (paratope) that fits to one of the antigen epitopes become activated and proliferate. Following a period of proliferation, the B-cells acquire an effector function: they secrete their antigen receptors as antibody molecules which in turn eliminate the antigen. In the present paper we ignore the important facts that most B-cell responses require help from helper $\mathrm{T}$ cells.

Jerne proposed his network hypothesis in 1974. Network theory is an implication of the axiom that the (random) primary repertoire is complete (Jerne, 1984). Jerne argued that the V-regions of lymphocyte receptors should also be recognized by other receptor molecules (paratopes). The antigenic determinants provided by a receptor molecule are called idiotypes (Jerne, 1974). The interaction between two receptor molecules (i.e. a paratope seeing an idiotype) is an idiotypic interaction. The set of such interactions defines the immune network. Via their idiotypic anti-idiotypic interactions, different clones should thus be able to interact in either a stimulatory or a suppressive manner. The existence of network interactions, i.e. of idiotypic interactions, has since been proved with numerous experimental data (Jerne, 1984). Moreover the possibility that the functioning of immune systems is regulated by network interactions seems intriguing. Several authors have suggested that immune networks may acount for: (1) the maintenance of steady states (Jerne, 1974; Cooper et al., 1984); (2) the control of proliferation (Jerne, 1974; Cooper et al., 1984); (3) the development of immunological memory (i.e. immunity, see Jerne, 1974); (4) low zone tolerance (Jerne, 1974); (5) self-non-self discrimination (Hoffmann, 1975; Jerne, 1984; Holmberg et al., 1986). Similarly, idiotypic networks have aroused considerable theoretical interest (Richter, 1975; 1978; Hoffmann, 1975; 1979; 1980; 1982; Hiernaux, 1977; Seghers, 1979; Gunther and Hoffmann, 1982; Fey et al., 1984; Fey and Eichmann, 1985; Kaufman et al., 1985; Kaufman and Thomas, 1987; Farmer et al., 1986; De Boer, 1988; Segel and Perelson, 1988; Perelson, 1988).

Jerne (1974) visualized the immune response as the "escape from suppression": in the absence of antigen (i.e. in the virgin state) network interactions are suppressive. According to Jerne (1974) this would guarantee the existence of a stable virgin state; see however De Boer and Hogeweg (in press). Antigens, e.g. viruses, perturb the suppressed state of the network, which results in proliferation and antibody production. For $\mathrm{T}$ cells similar "escape from suppression" models were proposed (based on the analyses of limiting dilution curves) (Cooper et al., 1984; Fey et al., 1984; Fey and Eichmann, 1985).

Hoffmann $(1975 ; 1978 ; 1980)$ developed a 2-D model (the "plus-minus" model) of the idiotypic network. This model incorporates the very important assumption that idiotypic interactions are symmetric, i.e. if clone $X_{i}$ recognizes $X_{j}, X_{j}$ should also recognize $X_{i}$. If recognition corresponds to complementary 
matching, an idiotypic interaction should indeed be symmetric. Moreover, cells are activated by receptor crosslinking (Hoffmann, 1980), which is a process that cannot descriminate between paratope and idiotype. Additionally a considerable amount of experimental evidence favours the idea that idiotypic interactions are symmetric (Hoffmann, 1980; Jerne, 1984). However, although Hoffmann's (1975) original suggestion that idiotypic interactions are symmetric is very attractive, it has been followed by only few authors (Fey et al., 1984; Fey and Eichmann, 1985; Jerne, 1984). Note that symmetric network theory disposes of the distinction between paratope and idiotype; instead it regards V-regions as "sticky ends" (Hoffmann, 1980).

Here we adopt the symmetric network theory. However we abandon the "escape from suppression" hypothesis: in our model we assume that the virgin state is devoid of (suppressive) network interactions. Such a model accounts for a (stable) virgin state maintained solely by the influx and turnover of newborn calls. Furthermore, we think that the most important role that suppression could play in regulating immune reactions is the control of excessive proliferation. This requires strong suppressive interactions when lymphocyte populations are large, and not, as "escape from suppression" implies, in the virgin state. We therefore assume that it is the large anti-idiotypic populations that are suppressive.

Here we investigate the effect that idiotypic interactions can have on the (normal) proliferative immune response to external (i.e. non-idiotypic) antigens. The effect of idiotypic interactions is studied as a function of (1) the affinity of the idiotypic interaction and (2) the number of idiotypic interactions (i.e. the size and connectance of the network). We thus require a model that incorporates: (1) influx and efflux of newborn cells; (2) symmetric stimulatory and suppressive idiotypic interactions; (3) affinity; (4) interaction with external antigens. None of the previous models described in the literature adequately incorporates these requirements; we therefore develop a new model.

2. Previous Models. Hoffmann $(1982 ; 1987)$ reviewing the previous idiotypic network models concluded that most of the models were inadequate because they: (1) were asymmetric; (2) lacked influx or efflux; (3) ignore clonal selection; (4) could not fulfil the Unpredictability Axiom. The Unpredictability Axiom states that the strength (i.e. affinity) of the idiotypic interactions must vary considerably, and that results obtained with network models should be robust to small variations in the interaction strength. In this review, Hoffmann however does not engage in a critical discussion of the symmetric network models put forward by him $(1975 ; 1979 ; 1980)$ and by Gunther and Hoffmann (1982). These models nevertheless have a number of inadequacies.

The Hoffmann models consists of two complementary clones, $X_{+}$and $X_{-}$; idiotypic interactions induce proliferation $\left(k_{1}\right)$ and suppression by $\operatorname{IgM}\left(k_{2}\right)$ 
and by $\operatorname{IgG}\left(k_{3}\right)$. The cells have a turnover rate $k_{4}$ and appear at a rate $S$ from thymus or bone marrow:

$$
\mathrm{d} X_{+} / \mathrm{d} t=X_{+} \cdot\left(k_{1} \cdot X_{-} \cdot E_{1}-k_{2} \cdot X_{-} \cdot E_{2}-k_{3} \cdot X_{-}^{2} \cdot E_{3}-k_{4}\right)+S .
$$

The "effectivity" $\left(E_{i}\right)$ of the idiotypic interactions is determined by the concentration of antigen specific (T cell) factors of either the "+" and the "-" specificity:

$$
E_{i}=1 /\left(1+\left(X_{+} \cdot X_{-} / C_{i}\right)^{N_{i}}\right), i=1,2,3,
$$

for which (Hoffmann, 1979):

$$
N_{1}=1, N_{2}=2, N_{3}=2 .
$$

The saturation or effectivity terms of these models incorporate the product of the idiotypic and anti-idiotypic $\left(X_{+} \cdot X_{-}\right)$population as a "whole". This product is large, i.e. the terms saturize, if (1) both populations are large, and (2) one of the two populations $\left(X_{+}\right.$or $X_{-}$) is sufficiently large. If this product is large, the $X_{+}$population proliferates at a rate $k_{1} \cdot C_{1}$ whatever the actual size of the $X_{+}$population. Similarly, IgM suppression approximates zero in all circumstances in which this product is large. Only IgG suppression depends on the $X_{+} / X_{-}$ratio. Per individual cell, these terms thus allow for infinite proliferation or suppression rates. By contrast, the saturation or efficiency of an idiotypic interaction between populations should remain interpretable at the level of the individual cells that comprise these populations. Indeed, the physiological constraints of individual cells (e.g. a minimum time span required for cell division) suffice for defining more realistic saturation effects. Moreover, the "global" effectivity terms of the Hoffmann models severely hamper the extension of these models with external antigen and/or additional lymphocyte clones.

The Hoffmann models omit affinity and external antigen. Incorporation of affinity is relatively easy and enables us to investigate whether these models fulfil the Unpredictability Axiom. It appears that the existence of four stable states, as described by Hoffmann (1979) and Gunther and Hoffmann (1982), requires a high affinity interaction. If affinity is lowered the Hoffmann (1979) model loses the two immune states, whereas the model of Gunther and Hoffmann (1982) loses the suppressed state and has only one immune state. The extension of the Hoffmann models with antigen is, by contrast, very complex because of the global nature of the efficiency terms. Stimulation with antigen should increase the production of $\mathrm{T}$ cell factors, and should therefore block the idiotypic interaction between the plus and minus clone.

We have tested a variety of implementations of external antigens in the Hoffmann (1979) model. These antigens grow exponentially and are eliminated 
by the IgM and IgG terms. This however failed to generate the classical picture of an immune response: proliferation followed by antigen regression, and finally settlement in an immune state. Depending on its initial size and/or its growth rate, antigen is either immediately eliminated or expands infinitely. Importantly, in our experiments with these models, the system never ended up in the suppressed state if we started with "physiological" initial conditions (i.e. in the virgin or immune state with or without antigen). Thus Hoffmann's suppressed state is "unattainable". We conclude that although the Hoffmann models define "escape from suppression" systems that account for switching to immune states and from IgM to IgG, the models are inadequate for investigating the role of idiotypic interactions in proliferative immune reactions to antigens.

3. A New Network Model. In our model we make two important choices: (1) idiotypic interactions are symmetric; (2) all populations are identical except for the antigen receptor (i.e. the idiotype). We choose for symmetry because it seems the most minimal (and, in our opinion, most likely) implementation of a recognition interaction based on complementary matching and receptor crosslinking. Secondly, we only consider one type of lymphocyte, i.e. we do not differentiate between "helper" and "suppressor" interactions, because: (1) idiotypic network theory was originally (Jerne, 1974) defined for identical clones of B-lymphocytes; (2) symmetric network theory (Hoffmann, 1979; 1980; Gunther and Hoffmann, 1982) has demonstrated that identical populations adopt "helper" or "suppressor" functions (i.e. phenotypes) depending on the idiotypic circumstances.

Although these two choices enable us to define a simple and attractive model of the (possibly) very complex idiotypic network, they necessitate another (third) choice: the distribution of stimulatory and inhibitory interactions. Idiotypic interactions are empirically known to be both positive and negative. During an immune reaction the responding idiotype can evoke the production of anti-idiotypic antibodies (Cosenza, 1976; Cerny, 1982). This is a positive interaction similar to a proliferative response to antigen. Administration of anti-idiotypic antibodies can both enhance (Eichmann and Rajewsky, 1975; Vakil and Kearny, 1986; Bernabe et al., 1981) and suppress (Hardt et al., 1972; Eichmann, 1974; Vakil and Kearny, 1986) the idiotypic immune response. Because all populations are identical in our model, and because idiotypic are symmetric, we have no a priori basis to discriminate between stimulatory and inhibitory interactions. The effect of an idiotypic interaction can therefore only depend on the (temporal) idiotypic circumstances, i.e. on the concentration of idiotype and anti-idiotype, and on the affinity of the interaction.

In contrast to what "escape from suppression" (Jerne, 1974) implies, we assume that idiotypic interactions are suppressive when the concentrations of 
anti-idiotype are large. In accordance with our previous work on proliferative immune reactions (De Boer and Hogeweg, 1986; 1987a,b) we thus assume that populations have to enlarge (by proliferation) before they can have a significant (here suppressive) effect. Idiotypic interactions are therefore absent or low if the anti-idiotypic populations are small, e.g. in the virgin state. In between, i.e. for intermediate-sized anti-idiotypic populations, interactions are stimulatory. In fact, this "proliferation before suppression" assumption corresponds to the widely accepted idea that the immune response is most vigorous at intermediate antigen concentrations. We, nevertheless, test the robustness of our results for our "proliferation before suppression" assumption by analysing an "escape from suppression" version of our model.

Clones have a constant influx $\left(S_{i}\right)$ of cells from the bone marrow. Cells have a constant turnover rate $(D)$. Differences between long-lived memory cells and short-lived effectors are thus deliberately ignored. Affinity is a parameter ranging between zero (no interaction) and one (maximum interaction). We ignore the possibility of an affinity threshold, i.e. a minimum affinity required for possible interaction. A clone interacting with a number of clones, interacts with the sum of the anti-idiotypic interactions ( $\alpha I d=$ anti-Idiotype). One specific immune network can now be defined completely by an affinity matrix A. $\mathbf{A}_{i j}$ specifies the affinity between $X_{i}$ and $X_{j}$. Symmetry in our model simply means $\mathbf{A}_{i j}=\mathbf{A}_{j i}$. Clones never recognize themselves (all $\mathbf{A}_{i i}=0$ ).

For external antigens we consider viruses $\left(V r_{i}\right)$ that grow exponentially. We assume, for simplicity, that virus elimination is identical to anti-idiotypic suppression. The interactions among idiotypes are thus equivalent to the interactions with external antigens, which is the basic assumption of idiotypic network theory. The interaction with antigen is however asymmetric because a virus usually activates lymphocytes but cannot suppress them (the AIDS virus is an exception to this rule). For reasons of simplicity we assume that each clone can see only one virus, and that viruses are seen by only one clone; viruses are always seen with maximum affinity. A virus is numbered according to the number of the clone that recognizes it. (If viruses are seen by more clones similar results can be obtained.)

We propose the following model:

$$
\begin{gathered}
\alpha I d_{i}=\sum_{j=1}^{n} \mathbf{A}_{i j} \cdot X_{j} \\
F\left(X_{i}, V r_{i}, \alpha I d_{i}\right)=\frac{B \cdot X_{i} \cdot\left(V r_{i}+\alpha I d_{i}\right)^{M}}{K_{B}^{M}+\left(F \cdot X_{i}\right)^{M}+\left(V r_{i}+\alpha I d_{i}\right)^{M}}-\frac{C \cdot X_{i} \cdot \alpha I d_{i}^{M}}{K_{C}^{M}+\left(F \cdot X_{i}\right)^{M}+\alpha I d_{i}^{M}} \\
\frac{\mathrm{d} X_{i}}{\mathrm{~d} t}=S_{i}-D \cdot X_{i}+X_{i} \cdot F\left(X_{i}, V r_{i}, \alpha I d_{i}\right)
\end{gathered}
$$


and for the antigens,

$$
\frac{\mathrm{d} V r_{i}}{\mathrm{~d} t}=R \cdot V r_{i}-\frac{C \cdot V r_{i} \cdot X_{i}^{M}}{K_{C}^{M}+\left(F \cdot V r_{i}\right)^{M}+X_{i}^{M}}
$$

The interaction function $F\left(X_{i}, V r_{i}, \alpha I d_{i}\right)$ seems rather baroque. However, for $F=0$ and $M=1$ it simply specifies the difference between two conventional saturation functions (one for proliferation and one for suppresssion). Because we choose $K_{B} \ll K_{C}$ the proliferation term $(B)$ outweighs the suppression term $(C)$ at low population $\left(X_{j}\right)$ densities. By choosing $C \gg B$ (i.e. the maximum suppression rate $\gg$ maximum proliferation rate), we assume that the suppression term over-rules the proliferation term at high population densities. Suppression is thus dominant (cf. Richter, 1978). For minimalization reasons it is important to know that most of the results described in this paper can also be accounted for by simpler models, i.e. $F=0$ and/or $M=1$ (see Fig. 1).

The model incorporates a "buffering" term $(F)$, see e.g. Richter (1978), in order to avoid that the interaction with $X_{i}$ cells can become independent of the size of $X_{i}$. This would lead to unrealistic model behaviour (especially in high dimensional networks). By choosing $F \ll 1$ we make these functions follow conventional saturation kinetics whenever $X_{i}$ populations are small. At the level of individual cells $F<1$ means that one $X_{j}$ cell can stimulate or suppress several $X_{i}$ cells.

Parameters. We exclude long-lived memory cells from our model: all cells live about 5 days $\left(D=0.2\right.$ days $\left.^{-1}\right)$. If life-long memory occurs it can thus only be caused by a state switch of the network. An influx of about $S_{i} \approx 20$ cells per day per clone thus generates a virgin clone size of $S_{i} / D=100$ cells. This is a reasonable estimate, De Boer and Hogeweg (1987a). A normal proliferation cycle takes about $16 \mathrm{hr}$ : a continuous increase of about 0.5 cells per cell fits this estimate. The maximum per cell increase is $B-D ; B$ should therefore equal 0.7 days $^{-1}$. Suppression is dominant (i.e. $C \gg B$ ); we take $C=25$ days $^{-1}$. The suppression threshold is higher than the proliferation threshold (i.e. $K_{B} \gg K_{C}$ ): $K_{B}=10^{3}$ and $K_{C}=10^{6}$. At low population densities, the effect of buffering should be negligible: $F=0.01$. The virus has a doubling time similar to the lymphocyte clones: $R=0.5$ days $^{-1}$. Unless explicitly stated otherwise, we use $M=2$ throughout.

In Fig. 1 we analyse the interaction between $X_{i}(i=1)$ and $X_{j}(j=2)$ for various interaction functions' $\left(F\left(X_{i}, V r_{i}, \alpha I d_{i}\right)\right.$, i.e. for $M=1, F=0$; for $F=0.01$ and/or $M=2$ ). The shaded regions depict the respective $X_{1}^{\prime}>0$ zones, i.e. the regions in which proliferation dominates suppression. The line at the boundary of such a region is the $O$-isocline. The figure also shows several other $X_{1}$-isoclines (i.e. $10,100, \ldots, 10^{6}$ ). Figure 1a (i.e. $F=0, M=1$ ) has an 
extremely high $\left(>10^{6}\right)$ proliferation region: about $10^{8} X_{1}$ cells proliferate in response to only $10^{4} X_{2}$ cells (i.e. $10^{-4}$ stimulator cells per $X_{1}$ ). This is unrealistic. If we include buffering $(F=0.01$, Fig. $1 b)$ this artefact is eliminated: the $O$-isocline closes. The maximum (net) proliferation rate is now about $10^{4}$
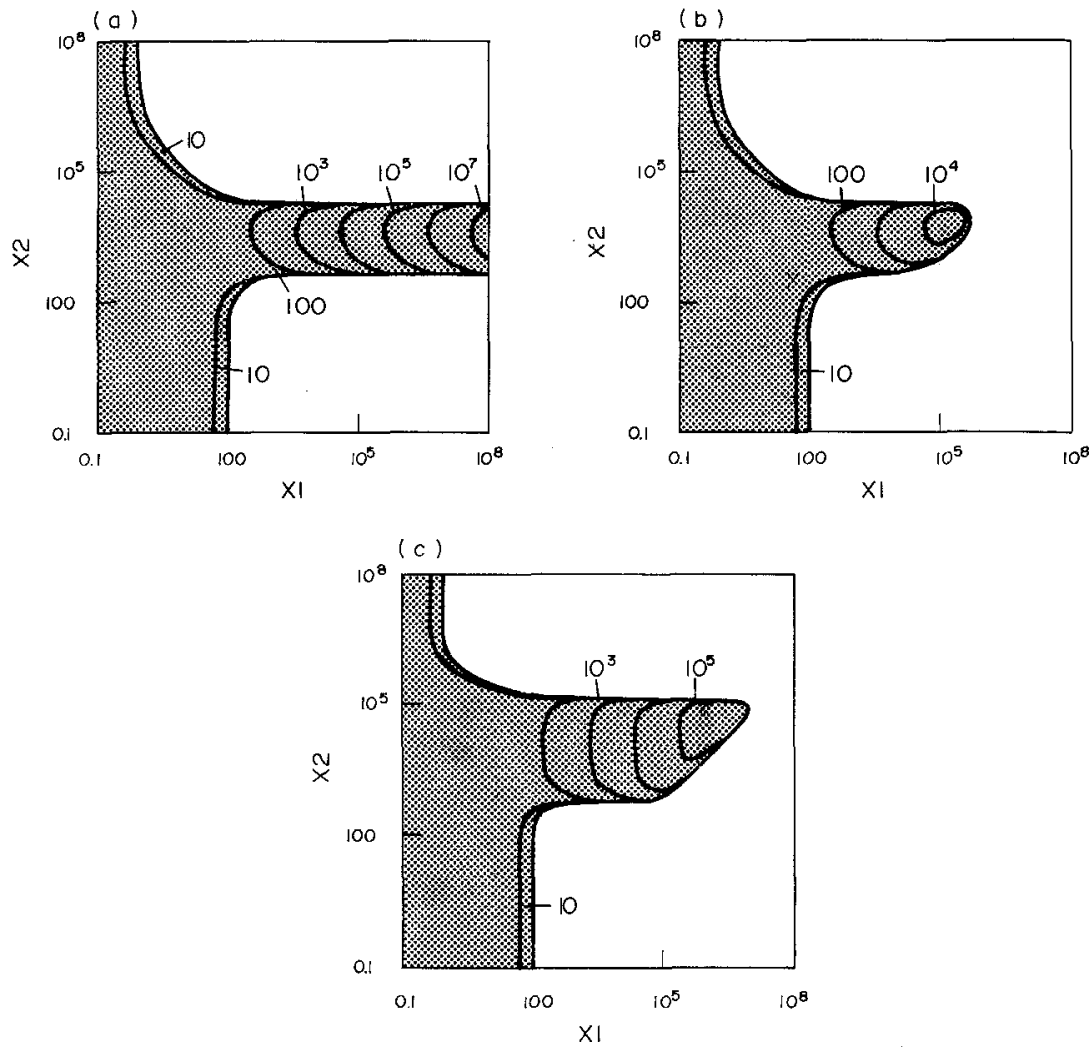

Figure 1 . The $0,10, \ldots, 10^{7}$ isoclines of the $X_{i}(i=1)$ clone in an $X_{1}-X_{2}$ state space, for three different versions of equation (2). (a) $F=0$ and $M=1$ (no buffering); (b) $F=0.01$ and $M=1$ (buffering); (c) $F=0.01$ and $M=2$ (buffering and a sigmoid dose response curve). The regions where proliferation dominates suppression $\left(X_{1}^{\prime}>0\right)$ are shaded.

cells per day (for an $X_{1}$ population of about $10^{5}$ cells). If $M>1$ the saturation functions in equation (2) become S-shaped (Hill functions). The isoclines for $M=2$, are shown in Fig. 1c: the shape of the $O$-isocline is somewhat different from that of Fig. $1 \mathrm{~b}(M=1)$. The proliferation region is wider: proliferation thus occurs for a larger range of $X_{2}$ values. In addition the maximum proliferation rate is higher. The switch between proliferation and suppression is thus more pronounced. The $O$-isoclines for even larger $M$ values (e.g. $M=5$ ) are very similar to those of Fig. 1c. The maximum proliferation rate is however 
ten times higher. We take $M=2$ and $F=0.01$ (Fig. 1c), because we do not want our results to depend on too extreme a saturation function.

The models are analysed by means of GRIND (De Boer, 1983); GRIND numerically searches for isoclines and performs numerical integration by means of ROW4A (Gottwald and Wanner, 1981).

\section{Results}

$A$ 2-D network. Addition of the $X_{2}^{\prime}=0$ isocline to Fig. 1c yields Fig. 2a. The $O$-isoclines of the two interacting idiotypes $\left(\mathbf{A}_{12}=1\right)$ intersect in five equilibria. The phase plot in Fig. 2b shows that three of these are stable (the stable states are encircled in Fig. 2a). The steady-states are (1) $V$ : the virgin state, both clones are at their respective $S / D$ values, a value too low for initiating an idiotypic interaction; (2) $I_{1}$ and $I_{2}$ : the immune states for $X_{1}$ and $X_{2}$ respectively. The immune states are asymmetric: in $I_{1}, X_{1}$ is immune, thus suppressing $X_{2}$, and in $I_{2}$ the situation is reversed. $X_{2}$ is suppressed in the $I_{1}$ immune state: it is far smaller than $X_{1}$ and cannot respond to its antigen $\left(V r_{2}\right)$ any further (see below). $X_{2}$ is nevertheless enlarged in the suppressed state; it is this enlargement of $X_{2}$ that sustains the immune state of $X_{1}$, Note that the enlargement of $X_{2}$ means that $X_{2}$ is still stimulated by $X_{1}$ (i.e. the interaction is not negative): we can however call $X_{2}$ suppressed because it can never proliferate again (e.g. to $V r_{2}$ ).
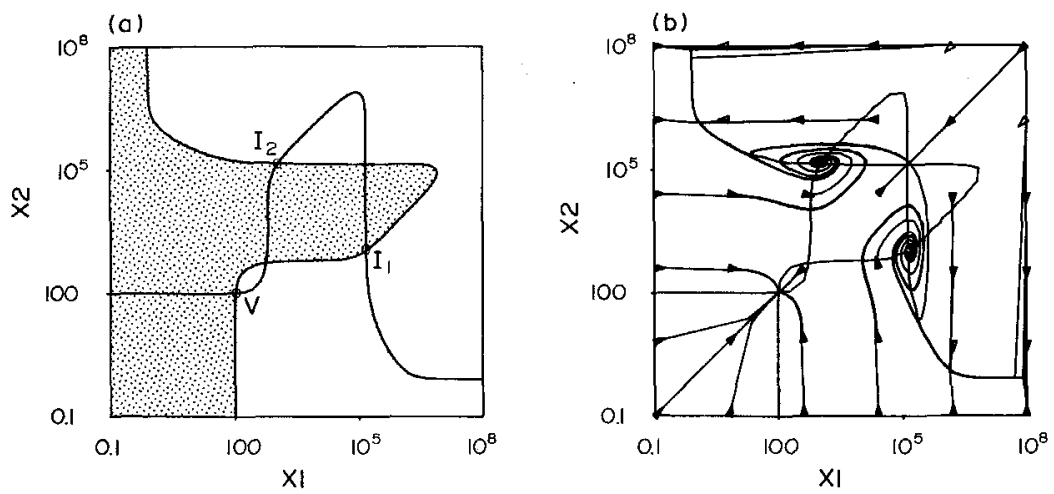

Figure 2. The $X_{1}$ and $X_{2}^{\prime}=0$ isoclines of a 2-D system $\left(\mathbf{A}_{12}=1\right)$. (a) The $X^{\prime}{ }_{1}>0$ region is shaded, and the stable equilibria are encircled. (b) The same $O$-isoclines in combination with trajectories starting at various points in the state space. The picture shows the oscillatory behaviour around the immune states.

Our $V, I_{1}$ and $I_{2}$ are equivalent to virgin, immune and anti-immune states of the Hoffmann $(1979 ; 1982)$ models. The present states are however generated with far simpler, and in our opinion better, saturation functions (note that even $F=0, M=1$ (equation (2)) yields these three states). The present model however lacks the stable suppressed states of the Hoffmann models. In the same 
region (i.e. both $X_{1}$ and $X_{2}$ large) however, the clones do suppress each other. In this model, concomitant suppression is not a stable situation. Other differences are the absence of interactions in our virgin state (Hoffmann virgin states are maintained by suppression) and the fact that our immune states are situated above the source/decay $(S / D$, i.e. no interaction) equilibrium, whereas those of the Hoffmann models are situated below it. In the Hoffmann models idiotypic interactions can only decrease population levels.

Affinity. The form of the $O$-isoclines and hence the existence of the steady states depend on the affinity of the $X_{1}-X_{2}$ idiotypic interaction $\left(\mathbf{A}_{12}\right)$. This is analysed in Fig. 3, which includes an affinity axis. The front of this cube is identical to Fig. $2\left(\mathbf{A}_{12}=1\right)$; the $V, I_{1}$ and $I_{2}$ state are again indicated. At the back $\left(\mathbf{A}_{12}=10^{-3}\right)$ the system only has a virgin state. Note that the position of the virgin state is hardly affected by the affinity. In the Hoffmann (1979) model, by contrast, the $X_{1}$ and $X_{2}$ population size in the virgin state decreases if the affinity increases (not shown).

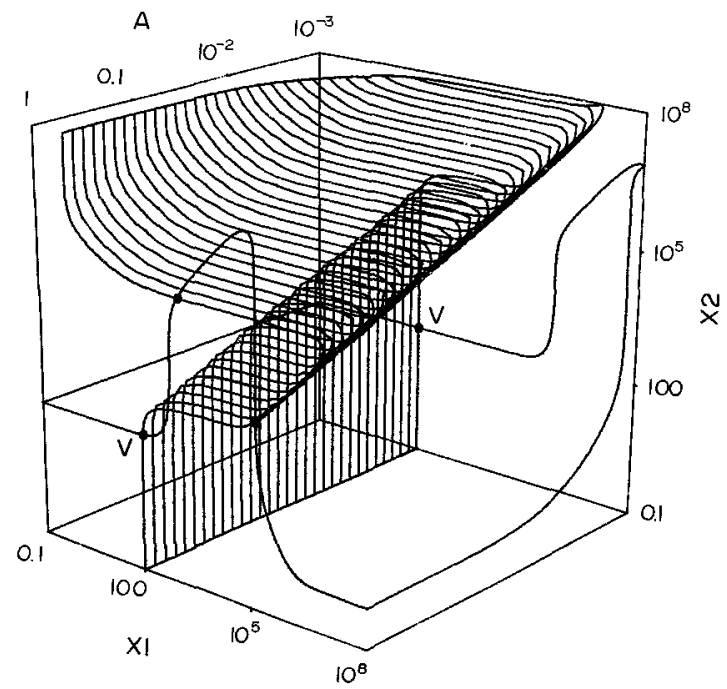

Figure 3. The $X_{1}$ and $X_{2}^{\prime}=0$ isoclines as a function of the affinity $\left(\mathbf{A}_{12}\right)$ of the idiotypic interaction. The front plane of this 3-D space is identical to Fig. 2 . The $X_{1}^{\prime}=0$ isocline plane is shaded, and the stable equilibria for $A_{12}=1$ and $A_{12}=10^{-3}$ are encircled.

We conclude from Fig. 3 that for immune states to exist the affinity must be sufficiently large. Clones with a low affinity interaction (e.g. $10^{-3}$ ), can never switch to an immune state, whatever their respective population densities. Thus although we omitted an affinity threshold from our model, i.e. a minimum affinity below which idiotypic interaction is impossible, the model nevertheless generates one. This emergent threshold is caused by the double 
saturation terms (i.e. by buffering): if $F=0$ (Fig. 1a) the $O$-isoclines run straight into infinity and hence always intersect. Dynamically, i.e. by numerical integration, these systems $(F=0)$ however never reach such high immune states.

Any specific 2-D network corresponds to a slice through the cube of Fig. 3. We concluded above that, depending on this affinity, the model yields different results: (1) if affinity is low $\left(\mathbf{A}_{12} \leqslant 0.006\right)$ the 2 -D system only has a virgin state (i.e. it cannot switch); (2) if affinity is sufficiently high $\left(\mathbf{A}_{12}>0.006\right)$ the system has virgin and immune states (i.e. it might be capable of switching). For low affinities, results are again qualitatively different. For instance, for $\mathbf{A}_{12}=0.01$, the system has one virgin and one immune state.

Antigen. First consider the interaction of one clone $\left(X_{1}\right)$ with its virus $\left(V r_{1}\right)$. This is the no network situation: all $\mathbf{A}$-matrix elements equal zero. The $O$-isoclines of this (1-D) system are shown in Fig. 4a. The $V r_{1}^{\prime}=0$ isocline is straight: the virus decreases whenever "its" lymphocyte clone exceeds a size of about $10^{5}$ cells. The $X_{1}^{\prime}=0$ isocline is more complex: at low $V r_{1}$ densities $X_{1}$ remains in its virgin state $(S / D)$. Higher $V r_{1}$ densities induce $X_{1}$ proliferation: the $O$-isocline bends to the right. At high $X_{1}$ densities, however, proliferation requires higher $V r_{1}$ densities (due to buffering): the $O$-isocline bends upwards. Every virus infection (trajectory) should start at the virgin state of $X_{1}$ : we start at $X_{1}=100$ and $V r_{1}=10$. The virus expands to about $10^{6}$ infected cells (Fig. 4), and meanwhile induces $X_{1}$ proliferation. As a result of this immune reaction, the virus regresses (i.e. dips to about 0.01 infected cells). Thus $X_{1}$ is no longer stimulated and declines: as a consequence the virus regrows. After a number of oscillations the system settles in a stable equilibrium with about $10^{3}$ infected cells $\left(V r_{1}\right)$ and $10^{5}$ immune cells $\left(X_{1}\right)$. We call this "virus dormancy": the virus remains present at a (harmless) low density, kept under control by a stimulated (enlarged) clone. This is a simple form of immunity: reintroduction of the same virus never leads to virus expansion because the system quickly returns to the dormancy equilibrium with hardly any clone proliferation. Note that many biotic viruses remain present in a similar dormant state (Bellanti, 1985). We conclude that virus dormancy can account for the "memory" phenomenon. Such an immune state does not require either long-lived memory cells or idiotypic interactions.

Note however that at various stages of the immune response the virus density was less than one infected cell. Although in a stochastic model this might correspond to a low but finite probability, we prefer to eliminate such (improbable) circumstances. Virus densities of less than one cell are therefore set to zero: this is virus elimination. If we do this in Fig. 4, the virus is rejected in the first cycle and the clone returns to its virgin state due to a lack of immune stimulation. Thus dormancy, and hence immunity, is lost. 

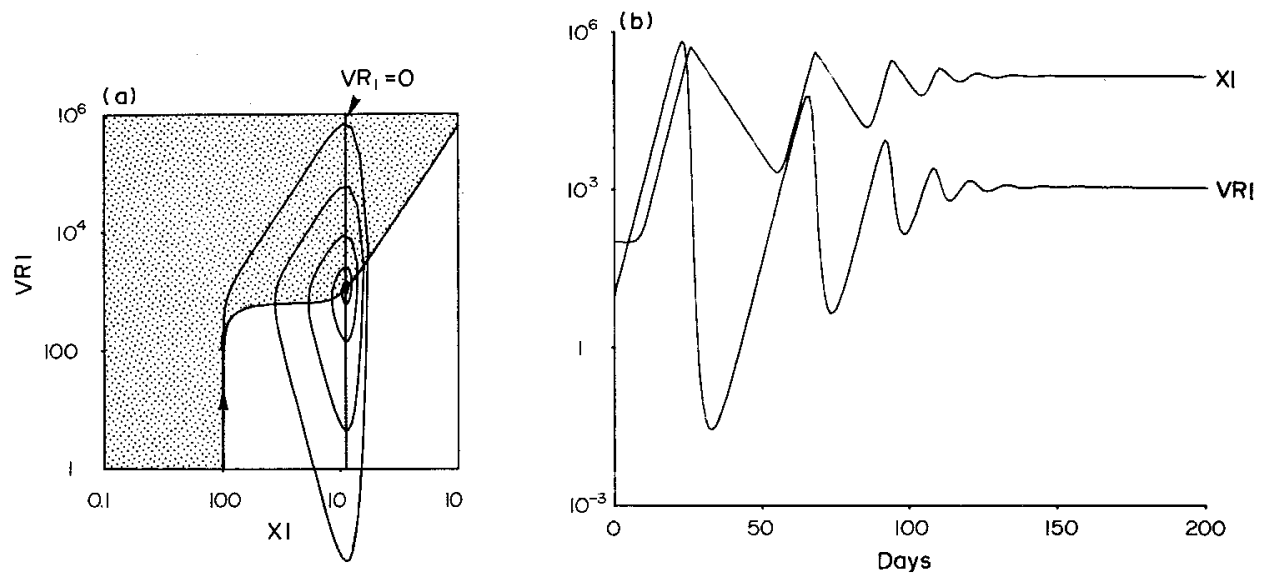

Figure 4. The interaction with $X_{1}$ and $V r_{1}$ without any network interaction. (a) Shows the $X_{1}^{\prime}=0$ and the $V r_{1}^{\prime}=0$ isocline (the $X_{1}^{\prime}>0$ region is again shaded). The trajectory depicts the immune response following the introduction of $V r_{1}=10$ in the virgin state of $X_{1}\left(X_{1}=100\right)$; the latter is marked by the circle. The response is highly oscillatory and settles in the stable intersect between the two isoclines. (b) The time plot of the same reaction.

Switching. We next study this virus in a 2-D network, e.g. that of Fig. 2 $\left(\mathbf{A}_{12}=1\right)$. Following the immune reaction to $V r_{1}$ this 2-D system switches from virgin to immune (i.e. to $I_{1}$, Fig. 5a). This is a "correct" switch: $X_{1}$ attains an immune state following an $X_{1}$ primary response to $V r_{1} ; X_{2}$ becomes suppressed. Experimental data also describe stable state switches that are based on differences in population sizes (Bernabe et al., 1981). Figure 5 b shows the time plot of this reaction: during the normal immune response the responding idiotype stimulates the production of the anti-idiotype. This is also described experimentally (Cosenza, 1976; Cerny, 1982; Möller and Fernandez, 1986). Around day 25, the virus is rejected and $X_{1}$ remains immune. Reintroduction of the same virus immediately leads to virus regression. Introduction of $V r_{2}$ in the $V$ state would of course lead to the $I_{2}$ state: the system is symmetric. Introduction of $V r_{2}$ in the $I_{1}$ state, i.e. after $V r_{1}$ rejection, leads to infinite growth of $V r_{2}$ in the absence of $X_{2}$ proliferation. $X_{2}$ really is suppressed.

This type of switching behaviour only occurs if $\mathbf{A}_{12} \geqslant 0.02$ (analysed dynamically by introducing viruses for a variety of different affinity values). Thus if affinities are distributed uniformly between one and zero, we expect $98 \%$ of all 2-D systems to switch to immunity after a primary immune response.

Static analysis. We analyse the $X_{1}, X_{2}, V r_{1}$ system statically in Fig. 6. At the back $\left(V r_{1}=1\right)$ Fig. $2 \mathrm{a}\left(\mathbf{A}_{12}=1\right)$, and at the bottom $\left(X_{2}=0.1\right)$ Fig. 4 a can be recognized. In the cube, the $V r_{1}^{\prime}=0$ isocline is straight: it depends on $X_{1}$ only. 

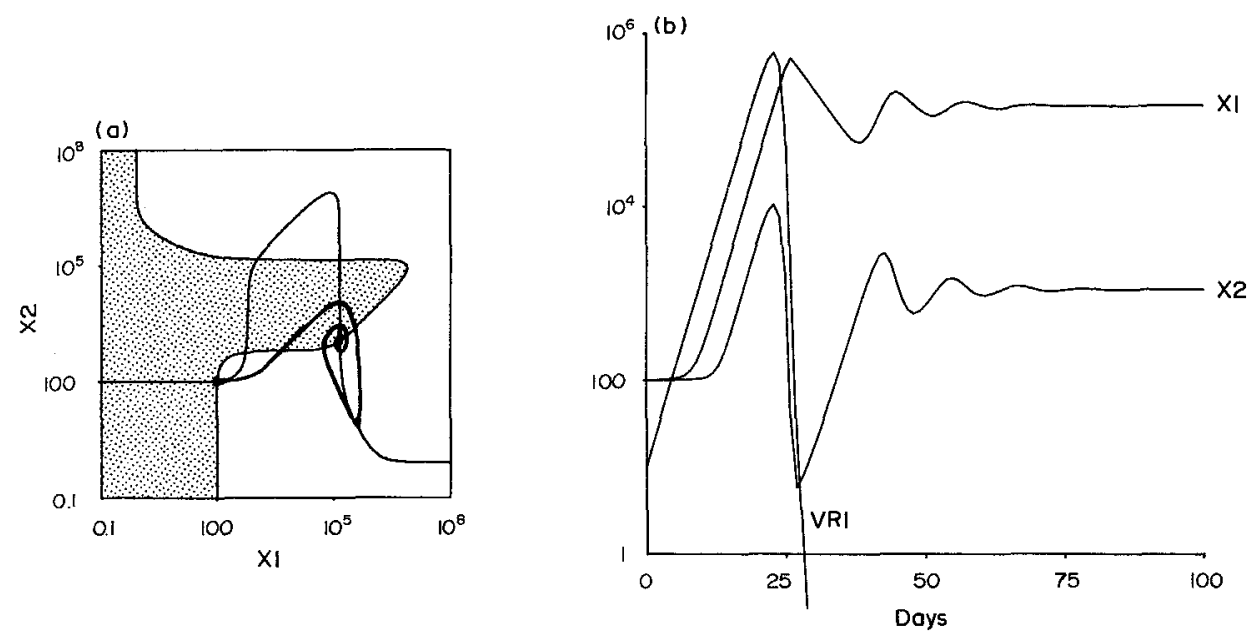

Figure 5. The interactions between $X_{1}, X_{2}$ and $V r_{1}$, i.e. a 2-D network with antigen. We again depict the 2-D state of Fig. $2 \mathrm{a}\left(\mathrm{A}_{12}=1\right)$, but we additionally project the immune responses to $V r_{1}$ in the figure (the fat line). This settles in the immunity equilibrium. The immune response is also shown in a time plot (b).

The $X_{2}^{\prime}=0$ isocline is independent of $V r_{1}$, i.e. it remains identical to that in Fig. 2. The upper part of the $X_{1}$ isocline is also independent of $V r_{1}$; the lower part, by contrast, bends to higher $X_{1}$ values at high $V r_{1}$ values. $X_{1}$ can thus be further stimulated by $V r_{1}$. At the upper part of the isocline $X_{1}$ is suppressed: an increase of $V r_{1}$ cannot influence the position of the $X_{1}$ steady-state. By contrast in the immune state $\left(I_{1}\right), X_{1}$ is enlarged and it is still capable of further expansion. Infinite $V r_{1}$ values allow for infinite $X_{1}$ populations. An $X_{1}$ population in the $I_{2}$ state however remains constant whatever the $V r_{1}$ density. Moreover the $X_{1}$ population in the $I_{2}$ state is too small to cause $V r_{1}$ regression: it is situated left of the $V r_{1}^{\prime}=0$ isocline. We conclude that $X_{1}$ is "immune" in the $I_{1}$ state and "suppressed" in the $I_{2}$ state.

From the results presented hitherto we conclude that in $98 \%$ of all the cases (if affinity is distributed uniformly) a 2-D network accounts for immunity/ memory due to a "correct state switch" following a primary response. Moreover, the switching behaviour of this model is robust: even simpler models (i.e. those based on equation (2) for $F=0$ and/or $M=1$ ) perform the same switches (albeit for a somewhat smaller range of affinity values). The present results are thus not the mere consequence of the choice of the form of the saturation function.

Suppression. Data like that in Fig. 5b, i.e. oscillations in the network response to $V r_{1}$, are usually interpreted in the form of idiotypic suppression (Cosenza, 1976; Bona, 1982). It indeed seems as if the enlargement of the antiidiotypic clone $\left(X_{2}\right)$ is responsible for the decline of the idiotypic $\left(X_{1}\right)$ 


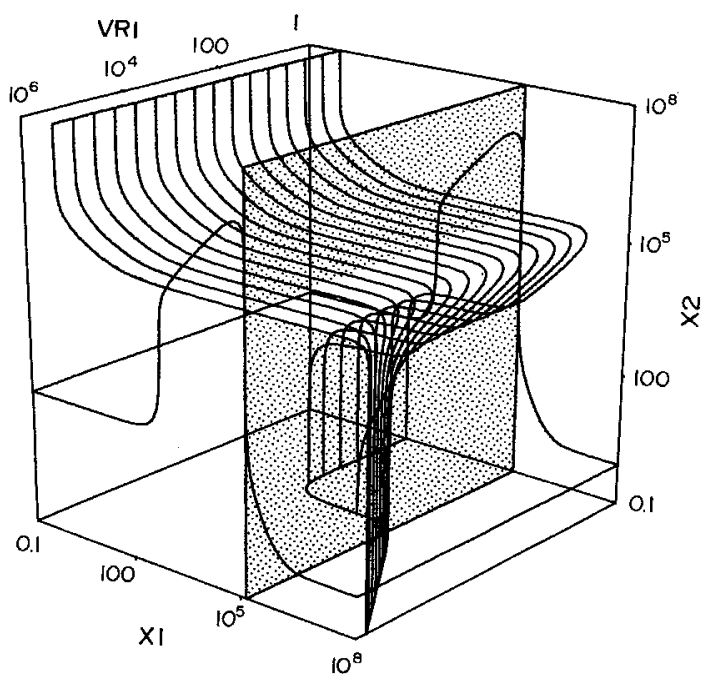

Figure 6. A static representation of the interaction between $X_{1}, X_{2}$ and $V r_{1}$. The $X_{1}^{\prime}=0, X_{2}^{\prime}=0$ and $V r_{1}^{\prime}=0$ isoclines in a $X_{1}, X_{2}, V r_{1}$ state space. The $X_{1}^{\prime}=0$ isocline plane is shaded. The $V r_{1}^{\prime}=0$ isocline is straight and situated at about $X_{1}=10^{5}$; the $X_{2}^{\prime}=0$ isocline is identical to that of Fig. $2\left(\mathbf{A}_{12}=1\right)$. The proliferation region of $X_{1}$ expands if $V r_{1}$ increases.

population. In the model however this is not the case: $X_{1}$ declines because the virus regresses, $X_{2}$ declines because $X_{1}$ declines. Experimentally, it was also shown that the decline of the responding idiotype $\left(X_{1}\right)$ is not caused by the anti-idiotype produced during the response (Möller and Fernandez, 1986). In the model an anti-idiotypic clone (here $X_{2}$ ) can never suppress the idiotypic clone $\left(X_{1}\right)$ that responds to antigen because $X_{2}$ is necessarily smaller than $X_{1}$. $X_{1}$ is always ahead because it responds first (to antigen). Thus it is never the anti-idiotype that (down) regulates the proliferation of the idiotypic clone $\left(X_{1}\right)$ but it is the proliferating idiotype that suppresses its anti-idiotypic "suppressors'. Proliferating clones "free" themselves of idiotypic interactions.

Conversely, suppression (proliferation regulation) does arise in these models if the system is (experimentally) manipulated by the introduction of antiidiotype $\left(X_{2}\right)$ prior to the introduction of the antigen $\left(V r_{1}\right)$. Such a procedure gives the anti-idiotype an advantage over the idiotype, and the system moves toward the $I_{2}$ state (in which $X_{1}$ is suppressed). In the absence of such experimental manipulations, i.e. in normal networks, this cannot happen.

Suppression, i.e. down regulation of the proliferating idiotype, is also absent from the models based on simpler interaction functions (i.e. $F=0$ and/or $M=1$ ). The absence of proliferation regulation is therefore a robust property of symmetric networks. Moreover, without experimental manipulation, the suppressed state of the (symmetric) Hoffmann models is also "unattainable". 
Furthermore, asymmetric versions of our model can (sometimes) account for suppression (i.e. if $\mathbf{A}_{21} \gg \mathbf{A}_{12}$ ). We conclude that symmetric networks fail to account for the control of proliferation (i.e. for suppression). This is a counterintuitive result because: (1) the model incorporates strong negative (suppressive) interactions $(C \gg B)$; (2) suppression is most intense at high population densities (i.e. after proliferation).

Escape from suppression. In order to test whether the absence of suppression hinges upon our "proliferation before suppression" assumption, we define an "escape from suppression" model (Jerne, 1974). According to this model idiotypic interactions are basically suppressive; antigen perturbs this suppressed state, thus allowing the clone to proliferate. This means that we have to replace equation (2). In equation ( $\left.2^{\prime}\right)$ anti-idiotype $\left(\alpha I d_{1}\right)$ no longer induces proliferation (i.e. $\alpha I d_{i}$ is absent from the proliferation term); anti-idiotypic suppression, by contrast, has remained identical. Antigen induces proliferation (as it does in equation (2)), and, additionally, reduces the rate of anti-idiotypic suppression (if $V r_{i} \approx K_{C}$ ):

$F\left(X_{i}, V r_{i}, \alpha I d_{i}\right)=\frac{B \cdot X_{i} \cdot V r_{i}^{M}}{K_{B}^{M}+\left(F \cdot X_{i}\right)^{M}+V r_{i}^{M}}-\frac{C \cdot X_{i} \cdot \alpha I d_{i}^{M}}{K_{C}^{M}+\left(F \cdot X_{i}\right)^{M}+V r_{i}^{M}+\alpha I d_{i}^{M}}$.

In the "virgin" state $\left(X_{i} \approx 100\right)$ idiotypic interactions have to be suppressive; we therefore set $K_{C}=100$ in equation ( $2^{\prime}$ ). Virus elimination (equation (4)) is however kept identical between the two models (i.e. for internal equivalence reasons (Irvine and Savageau, 1985); $K_{C}$ in equation (4) is kept at $10^{6}$ ). All other parameters can remain the same. Note that in this escape from suppression model $K_{C} \ll K_{B}$; we thus first have suppression and then proliferation.

The $X_{1}-X_{2}$ state space of this model (for $\mathbf{A}_{12}=1$ ) has three equilibria (Fig. 7a); two of them $\left(V_{1}\right.$ and $\left.V_{2}\right)$ are stable. Both correspond to some sort of virgin state, i.e. they are reached in the absence of antigen. Which of the two is reached depends on the respective influx parameters $\left(S_{i}\right)$. We thus have to consider two different initial states for the introduction of antigen. For a wide range of affinity values (Fig. 7b) the system either has one virgin state, with hardly any idiotypic interactions, or the two virgin states described above. Immune states (situated at elevated population sizes) are absent.

Introduction of a virus (here $V r_{1}$ ) in the two virgin states indeed leads to escape from suppression (Fig. 8). If $V r_{1}$ is introduced in the $V_{1}$ state (Fig. 8a), $X_{1}$ starts to proliferate when the virus is sufficiently large, i.e. after about 10 days. This increase of the idiotype further suppresses the anti-idiotype $\left(X_{2}\right.$ further decreases). Thus, again, the proliferating idiotype suppresses its antiidiotypic suppressors and "escapes" from further regulation. Following virus 

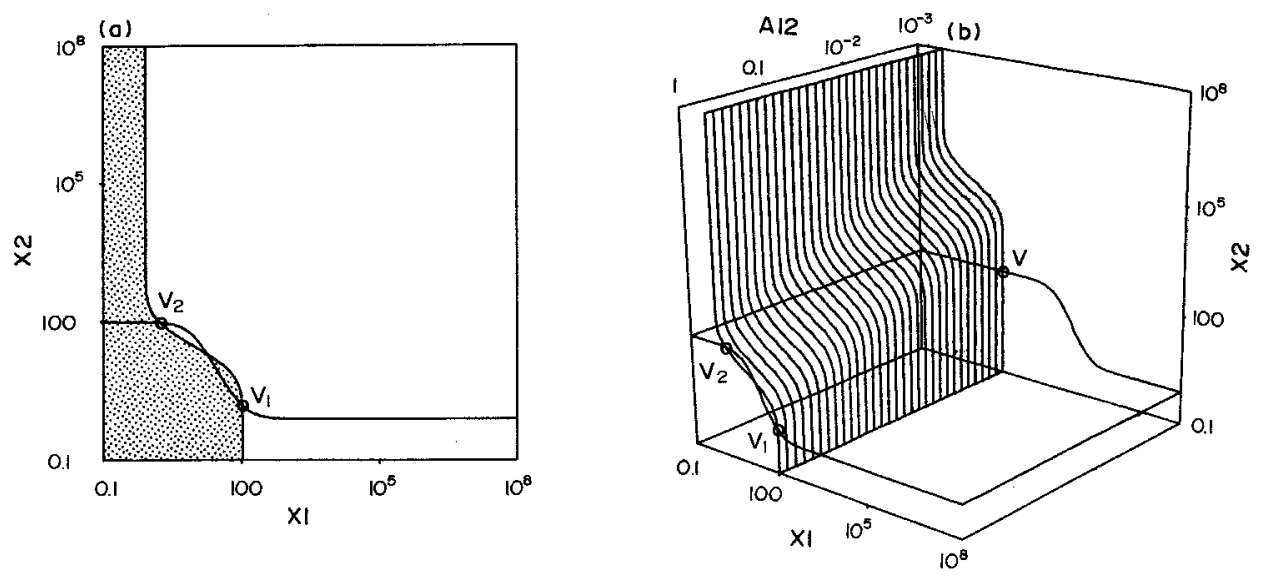

Figure 7. An "escape from suppression" model. The $X_{1}^{\prime}=0$ and $X_{2}^{\prime}=0$ isoclines are displayed in the $X_{1}, X_{2}$ state space for the affinity $\mathbf{A}_{12}=1$ (a), and as a function of affinity (b). Stable equilibria are encircled; the $X_{1}^{\prime}>0$ region is shaded. The system has one or two virgin states but no immune states.

rejection the system returns to the $V_{1}$ state. We conclude that both immunity and proliferation regulation are absent from this system. Similar behaviour is obtained when $V r_{1}$ is introduced in the $V_{2}$ state (Fig. 8b). Because the idiotype starts at a smaller population level the immune reaction is somewhat (but minimally) delayed. Following idiotypic $\left(X_{1}\right)$ proliferation the anti-idiotype again becomes (further) suppressed. Thus again proliferation regulation by the anti-idiotype is impossible. Following virus rejection the system does however switch from $V_{2}$ to $V_{1}$; this gives the idiotype a small advantage (compare Fig. 8a with 8 b).

This switch from $V_{2}$ to $V_{1}$ is a form of immunological memory. However, it is rather peculiar because: (1) it only develops erratically, i.e. if $S_{1}<S_{2} ;(2)$ if $X_{1}$ is called immune in the $V_{1}$ state, $X_{2}$ should be called immune in the (virgin!) $V_{2}$ state. Hence, immunity occurs in escape from suppression models, but only erratically and/or in the absence of the corresponding antigen. Moreover, note that this escape from suppression network fails to account for the production of anti-idiotypes during an immune response (Cosenza, 1976; Cerny, 1982; Möller and Fernandez, 1986). During the immune reaction the idiotype escapes but the anti-idiotype becomes further suppressed. The anti-idiotype is thus eliminated and the system no longer behaves as a network.

We conclude that in a symmetric system, escape from suppression has two meanings: (1) stimulation by antigen cancels the suppressive anti-idiotypic interaction; (2) the anti-idiotypic clones are suppressed to a level near elimination. Secondly, we conclude that our "proliferation before suppression" model behaves in a superior way, and, most importantly, that the absence of 

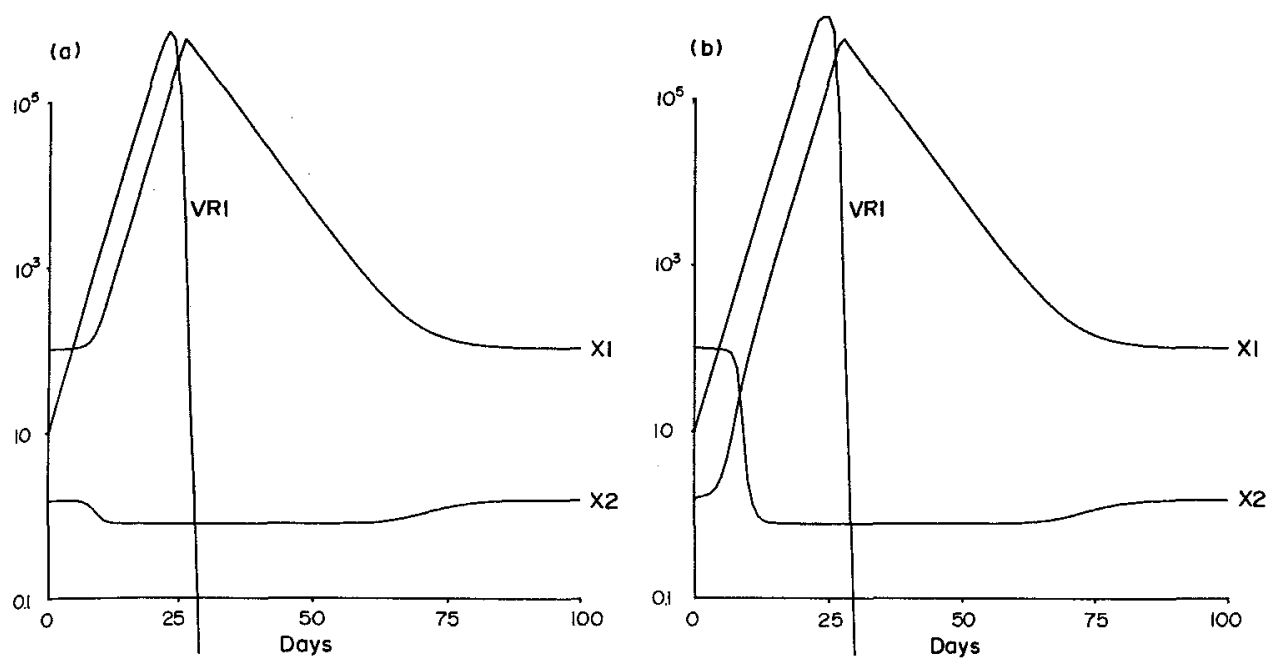

Figure 8. Immune responses in the escape of suppression model. In each of the two virgin states ((a) $V_{1}$; (b) $V_{2}$ ) $V r_{1}$ is introduced in a dose of 10 infected cells. In both cases the anti-idiotypic clone $\left(X_{2}\right)$ becomes further suppressed. The system always returns to $V_{1}$.

suppression in such a model does not hinge upon the order of proliferation and suppression.

3-D system. We therefore proceed with the analysis of our original [equations (1)-(4), $K_{B}<K_{C}$ ) model. Expanding that 2-D model into three dimensions is technically easy. The analysis of a 3-D model is however much more complex because we now have to consider a number of affinity parameters, each of which is expected to vary widely. Consider the conventional scheme of the immune networks (i.e. $X_{1}$ sees antigen, $X_{2}$ sees $X_{1}$, $X_{3}$ sees $X_{2}$, and so on). Thus in our symmetric A-matrix: $\mathbf{A}_{12}=\mathbf{A}_{21}>0$ and $\mathbf{A}_{23}=\mathbf{A}_{32}>0$, and all other $\mathbf{A}$ elements equal zero. Qualitatively we thus have to consider two affinity parameters. Additionally however, a 3-D network allows two qualitatively different antigens: (1) $V r_{1}$ an antigen seen by a clone with one connection to the network; (2) $V r_{2}$ an antigen seen by $X_{2}$ with two connections (i.e. to $X_{1}$ and $\left.X_{3}\right) . V r_{3}$ is qualitatively identical to $V r_{1}$ : the system is symmetric in $X_{2}$ ).

We have analysed this 3-D system by the introduction of these two antigens for all different combinations of the two affinity parameters $\left(\mathbf{A}_{12}\right.$ and $\left.\mathbf{A}_{23}\right)$. We thus attained nine qualitatively different stable states. In the absence of antigen the systems always remains in the 3-D virgin state. Antigenic stimulation yields 2-D immune states (as described above) if one of the affinities is sufficiently low. The weakly connected clone remains virgin, the other two switch to imune or suppressed. If both affinities are sufficiently high, the system switches to states 
where either two clones are immune and one is suppressed, or to states where two clones are suppressed by one immune clone.

Most of these switches correspond to "correct" immunity phenomena. In fact, depending on the circumstances, the additional idiotypic interaction in the 3-D system can both facilitate or inhibit the correct switching behaviour. Inhibition is more common however. If $X_{1}$ is stimulated by antigen, and the interaction between $X_{2}$ and $X_{3}$ is much stronger than that between $X_{1}$ and $X_{2}$, the correct switching behaviour is disrupted; $X_{1}$ then generally returns to virgin. Proliferation regulation does not occur in 3-D systems: even clones with two idiotypic connections suppress both anti-idiotypic clones before these can become suppressive. We conclude that the results obtained with the 2-D network can largely be repeated with 3-D systems.

5-D network. The most straightforward extension of the above 3-D network is one in which $X_{1}$ is seen by two clones of level 2, which each in turn are seen by one clone of level 3. Thus $X_{1}$ is doubly connected (as $X_{2}$ was) to 2-D systems identical to those analysed above. The affinity matrix now has 4 symmetric connections, i.e. 8 non-zero elements. We distribute these values uniformly between one and zero (by a pseudo random number generator). We want to find out whether an idiotypic interaction between $X_{1}$ and $X_{2}$ (which accounts for correct switching in $98 \%$ of the cases), can be disturbed by the three additional idiotypic interactions of a 5-D network. We have tested 40 such systems. Because all randomly drawn $\mathbf{A}_{12}$ values exceeded 0.01 , we expect all 40 networks to switch to an $I_{1}$ state after the introduction of $V r_{1}$. Such a switch was indeed found for all 40 networks. The immune states of $X_{1}$ are somewhat lower because $X_{1}$ can now be suppressed by two clones of level $2 ; X_{1}$ immunity is thus less vigorous (i.e. may lead to virus dormancy instead of virus rejection).

However, if we connect this system randomly instead of according to the above scheme, results become different. We assign to $\mathbf{A}_{12}$ a random value between zero and one; additionally such values are assigned to three other randomly chosen connections. We again analysed 40 networks; again a switch to immunity of $X_{1}$ was expected for each of them. However we find only 32 such switches: in 8 cases $(20 \%) X_{1}$ returns to its virgin state. Thus the general occurrence of correct switching behaviour (i.e. immunity/memory) seems to require a specific structure of the network. It is not at all clear whether biotic immune networks fulfil these conditions. Switching can for instance be disturbed if $X_{1}$ is only connected to $X_{2}$, and if $X_{2}$ is suppressed due to other network interactions. Such "disturbance" already occurred in 3-D systems. We conclude that the expansion of the present model to a random high- $D$ network may reduce the model's capacity to switch, i.e. to generate immunity. We are currently working on this question. 


\section{Discussion.}

Thresholds. The most important decision that has to be made during the development of an idiotypic network model is how to distribute the positive and negative interactions (i.e. Jerne's, 1974, third dualism). In asymmetric models the (asymmetric) affinity matrix may define non-overlapping sets of positive and negative interactions (i.e. it defines a different distribution of affinities on each side of the diagonal). Thus each individual idiotypic antiidiotypic interaction has either a positive or negative sign. Farmer et al. (1986) represent paratopes and idiotypes by bit-patterns which yields such an asymmetric affinity matrix. The Richter (1978), the Hoffmann models (1979; 1982), and the present model instead define a switch in sign depending on the size of the anti-idiotypic clone.

Jerne's (1974) "escape from suppression" idea was followed by Richter (1978): his suppression threshold is lower than the proliferation threshold (his $B>D$ ). In the Richter "virgin state" however, network interactions are absent, i.e. the population densities are far below the suppression threshold (Hiernaux, 1977). In the Hoffmann models $(1979 ; 1982)$ the virgin state is maintained by suppression; the suppression terms switch off at high population densities (even in the "suppressed state"). Thus, the Hoffmann models are "escape from suppression" systems.

In this paper we chose the opposite (our $K_{B}<K_{C}$ ), i.e. idiotypic interactions are absent in the virgin state and only large clones evoke suppression. We think this makes most sense if the stimulation and suppression processes are considered at the level of individual cells. Stimulation, and hence proliferation, of a cell requires crosslinking of its receptors by anti-idiotypic antibody. An increase in anti-idiotypic antibody is thus expected to increase proliferation. Suppression can occur by various mechanisms: (1) the crosslinking process may become less efficient if anti-idiotypic antibody concentrations become too high (Goldstein, 1988); (2) massive release of antibodies by idiotypic plasma cells will neutralize anti-idiotypic antibodies by complex formation; (3) coating of cells with anti-idiotypic antibodies may initiate the complement cascade, and lead to cell lysis (Eichmann, 1974; Hoffmann, 1979). All these mechanisms probably require higher antibody concentrations than does the initial crosslinking process that leads to proliferation.

Parameter constraints. We choose the proliferation saturation constant $\left(K_{B}\right)$ larger $(10 \times)$ than the virgin population size in order to keep network interactions low in the virgin state. The choice of $M=2$, i.e. an S-shaped dose response curve, also facilitates the preservation of the virgin state: if $M=1$ the 2 -D virgin state only exists if $\mathbf{A}_{12} \leqslant 0.88$. Thus in a model with linear $(M=1)$ saturation function the switching range is somewhat smaller $\left(0.04 \leqslant \mathbf{A}_{12} \leqslant\right.$ $0.88)$. Because the virus grows relatively fast $(R=0.5)$, the immune reaction has 
to be quite vigorous (i.e. to bring about much proliferation). This facilitates the establishment of network interactions because clones easily grow above the threshold required for inducing the proliferation of an anti-idiotypic clone $\left(K_{B}\right)$. In addition, virus rejection is more severe following a vigorous immune reaction; this prevents dormancy development (De Boer and Hogeweg, 1986). High lymphocyte turnover rates $(D)$ increase the instability (i.e. the oscillations) and lead to increased virus dormancy development. The abundant switching behaviour of this model occurs however for various parameter settings (not shown).

Immunological memory. The fact that an immune network can account for immunological memory does not mean that the memory phenomena displayed by biotic immune systems are indeed generated by network state switches. The existence of long-lived memory cells (Jerne, 1984; Levy and Couthino, 1987) provides a very simple alternative explanation. An alternative memory cell that would account for immunity is a cell which by proliferation and differentiation becomes insensitive to network suppression (Cooper et al., 1984). Thus by adding this assumption, one can account for memory in an "escape from suppression" model. Immunity generated by memory cells is not stable; although these cells are long-lived, they decline slowly. The main difference between network immunity and the two forms of memory cell immunity is that the former is (actively) maintained by proliferation whereas the latter corresponds to a resting state. Thus experimental data describing the existence of small, resting, long-lived cells (Jerne, 1984; Levy and Couthino, 1987) support the memory cell alternative. Moreover the dynamics of (long-lived) memory cells can account for self-non-self discrimination (De Boer and Hogeweg, 1987a, 1987b). Nevertheless, immunity generated by state switching remains an intriguing possibility; the present results prove that if proliferation precedes suppression it is a feasible possibility.

Suppression. The fact that our symmetric model fails to account for the suppression of proliferating clones violates the most important role of the immune networks. If anti-idiotypic interactions were indeed able to control excessive responses this would be very important (e.g. in self-non-self discrimination). In our model however this is impossible. Note that suppression can be generated by "experimental" manipulation, e.g. by the introduction of large anti-idiotypic clones, but never evolves during a "physiological" immune reaction. Experimental data that thus demonstrate the existence of suppression therefore provide no evidence for a role for suppression in the regulation of immune reactions. We think that suppression will be absent from most, if not all, symmetric networks because a proliferating clone is always ahead and hence should always be able to suppress its anti- 
idiotypic suppressors long before these can become suppressive. Our present parameter setting, i.e. proliferation before suppression $\left(K_{B}<K_{C}\right)$ and dominant suppression $(C \gg B)$, should facilitate the suppression of large proliferating clones because the suppression interaction is most intense in this region. Moreover, if we make our model asymmetric (by defining an asymmetric affinity matrix) suppression sometimes occurs. We thus conclude that symmetric network theory seriously violates the role of anti-idiotypic suppression in the control of (excessive) proliferation. In our extensions of the Hoffmann models we reach the same conclusion because proliferating clones moving in the direction of the immune state (thus suppressing the antiidiotypic partners) never end up in the suppressed state. The absence of suppression in our model suggests that the model can be further simplified by the removal of the negative iteractions $(C>B)$. Indeed, an alternative model would be one in which suppression only reduces stimulation. This can easily be incorporated by setting $C=B$ : such a (2-D) model generates a very similar result (submitted).

Network dimensions. The results obtained with our 2-D network also occurred in our 3-D networks and in 5-D networks of a specific structure. Randomly connected 5-D networks however showed different results. This suggests that the present (low-D) results may depend strictly on the form and dimension of the network. Recent work (submitted) confirms this: the low-D results described here can hardly be repeated in high-D networks.

The Hoffmann models $(1975 ; 1979 ; 1980 ; 1982)$ assume that the $10^{7}-\mathrm{D}$ immune network comprises separate pairs of interacting clones (plus minus clones). Wikler et al. (1979) showed that antibodies of the third level (Ab3) are idiotypically related to the Ab1 level; Ab4 in turn is related to Ab2. These data suggest that the network is "flat" instead of "open-ended". Similarly, Jerne (1984) concludes that "one characteristic of the idiotypic network is the occurrence of pairs of antibodies, of preferred partners". If these interpretations are correct, the idiotypic network is a (1-D) stack of independent (2-D) systems. We think that such a flat system should not be called a network (at least all comparisons with e.g. neural networks break down). In addition, as Jerne (1984) also argues, it implies that anti-idiotypic interactions have to be totally different from immune reactions to non-network antigens (epitopes) because the latter involve several hundreds, instead of one, antibody molecules. Moreover, idiotypes can have as many as 40 antigenic determinants (idiotopes) (Novotny et al., 1987), and, secondly, anti-idiotypic clones obtained from immature immune systems do show a high connectance to other clones (Holmberg et al. 1984; 1986; Vakil and Kearny, 1986).

If the idiotypic network would indeed be nothing more than a collection of independent pair-wise complementary interactions, the conclusions of the 
present paper should be valid for the complete $10^{7}-D$ immune network. However, we recently (submitted) found that if the high-D idiotypic network is open-ended, i.e. is not organized in such monogamous pair-wise interactions, the sytem behaviour becomes non-sensical because of extensive percolation of idiotypic signals. Therefore, it is an interesting question to analyse which cellular interactions in large (possibly randomly connected) idiotypic networks might cause the functional reduction to the pair-wise idiotypic interactions described above.

The authors would like to thank Miss S. M. McNab for linguistic advice.

\section{REFERENCES}

Bellanti, J. A. 1985. Immunology III, pp. 283-305, London: Saunders.

Berek, C., G. M. Griffiths and C. Milstein. 1985. "Molecular Events During Maturation of the Immune Response to Oxazolone." Nature 316, 314-318.

Bernabe, R. R., A. Coutinho, C. Martinez-A and P. A. Cazenave. 1981. "Immune Networks. Frequencies of Antibody- and Idiotype-Producing B Cell Clones in Various Steady-States." J. Exp. Med. 154, 552-556.

Bona, C. A. 1982. "Inverse Fluctuations of Idiotypes and Anti-Idiotypes During the Immune Response." In Regulation of Immune Response Dynamics, C. DeLisi and J. R. J. Hiernaux (Eds), Vol. I, pp. 75-82. Boca Raton, FL: CRC Press.

Burnet, F. 1959. The Clonal Selection Theory of Acquired Immunity. Cambridge: Cambridge University Press.

Cerny, J. 1982. "The Role of Anti-Idiotypic T Cells in the Cyclical course of an Antibody Response." In Regulation of Immune Response Dynamics, C. DeLisi and J. R. J. Hiernaux (Eds), Vol. I, pp. 59-73. Boca Raton, FL: CRC Press.

Cooper, J., K. Eichmann, K. Fey, I. Melchers, M. M. Simon and H. U. Weltzien. 1984. "Network Regulation Among T Cells: Qualitative and Quantitative Studies on Suppression in the Non-Immune State." Immunol. Rev. 79, 63-86.

Cosenza, H. 1976. "Detection of Anti-Idiotypic Reactive Cells in the Response to Phosphorylcholine." Eur. J. Immunol. 6, 114-116.

De Boer, R. J. 1983. GRIND: Great Integrator Differential Equations. Bioinformatics Group, University of Utrecht, The Netherlands.

and P. Hogeweg. 1986. "Interactions Between Macrophages and T-Lymphocytes: Tumor Sneaking Through Intrinsic to Helper T Cell Dynamics." J. Theor. Biol. 120, 331-351.

and $-1987 \mathrm{a}$. "Immunological discrimination between self and non-self by precursor depletion and memory accumulation." J. Theor. Biol. 124, 343-369.

- and 1987b. "Self-non-self Discrimination due to Immunological Non-Linearities: The Analysis of a series of Models by Numerical Methods." IMA J. Math. Appl. Med. Biol.4, $1-32$.

- 1988. "Symmetric idiotypic networks: connectance and switching, stability, and suppression." In: Theoretical Immunology-Part Two. SFI Studies in the Science of Complexity, A. S. Perelson (Ed.), Vol. III, pp. 265-289. Reading, MA: Addison-Wesley.

and P. Hogeweg. 1989. "Stability of Symmetric Idiotypic Networks: A Critique of Hoffmann's Analysis." Bull. math. Biol. 51, 217-222.

- and - In press. "Unreasonable Implications of Reasonable Idiotypic Network Assumptions."

Early, P. H. Huang, M. Davis, K. Calame and L. Hood. 1980. "An Immunoglobulin Heavy Chain Variable Region Gene is Generated from Three Segments of DNA: $V_{H}, D$ and $J_{H} . " C e l l$ 19, 981-992. 
Eichmann, K. 1974. "Idiotypic Suppression. I. Influence of the Dose and of the Effector Functions of Anti-Idiotypic Antibody on the Production of an Idiotype." Eur. J. Immunol. 4, 296-302.

- and K. Rajewsky. 1975. "Induction of T and B Cell Immunity by anti-Idiotypic Antibody." Eur. J. Immunol. 5, 661-666.

Farmer, J. D., N. H. Packard and A. S. Perelson. 1986. "The Immune System, Adaptation, and Machine Learning." Physica 22D, 187-204.

Fey, K., M. M. Simon, I. Melchers and K. Eichmann. 1984. "Quantitative Estimates of Diversity, Degeneracy, and Connectivity in an Idiotypic Network Among T Cells." In The Biology of Idiotypes, M. I. Green and A. Nisonoff (Eds), pp. 261-277. New York: Plenum Press.

___ and K. Eichmann. 1985. "Cluster Formation in a Symmetrical Network: A Dynamical System for the Description of the Suppression Among Non-Immune T-Lymphocytes and its Application to the Effects of Immunization." J. Theor. Biol. 114, 615-640.

Goldstein, B. 1988. "Desensitization, Histamine Release and the Aggregation of IgE on Human Basophils". In: Theoretical Immunology-Part One. Studies in the Science of Complexity, A. S. Perelson (Ed.), Vol. II, pp. 3-40. Reading, MA: Addison-Wesley.

Gottwald, B. A. and G. Wanner. 1981. "A Reliable Rosenbrock Integrator for Stiff Differential Equations." Computing 26, 355-360.

Gunther, N. and G. W. Hoffmann. 1982. "Qualitative Dynamics of a Network Model of Regulation of the Immune System: A Rationale for the IgM to IgG Switch." J. Theor. Biol. 94, 815-855.

Hardt, D. A., A. L. Wang, L. L. Pawlak and A. Nisonoff. 1972. "Suppression of Idiotypic Specificities in Adult Mice by Administration of Anti-Idiotypic Antibody." J. Exp. Med. 135, 1293-1299.

Hiernaux, J. 1977. "Some Remarks on the Stability of the Idiotypic Network." Immunochem. 14, 733-739.

Hoffmann, G. W. 1975. "A Theory of Regulation and Self Non-Self Discrimination in an Immune Network." Eur. J. Immunol. 5, 638-647.

- 1979. "A Mathematical Model of the Stable States of a Network Theory of Self-Regulation." In Systems Theory in Immunology-Lecture Notes in Biomathematics, C. Brunei, G. Doria, G. Koch and R. Strom (Eds), Vol. 32, pp. 239-257. Berlin: Springer.

-1 1980. "On Network Theory and H-2 Restriction." In Contemporary Topics Immunobiology, N. L. Warner (Ed.), Vol. 11, pp. 185-226. New York: PLenum Press.

- 1982. "The Application of Stability Criteria in Evaluating Network Regulation Models." In Regulation of Immune Response Dynamics, C. DeLisi and J. R. J. Hiernaux (Eds), Vol. I, pp. 137-162. Boca Raton, FL: CRC Press.

. 1987. "A Critique of the Kaufman-Urbain-Thomas Immune System Network Theory." J. Theor. Biol. 129, 355-357.

Holmberg, D., S. Forsgen, F. Ivars and A. Coutinho. 1984. "Reactions Among IgM Antibodies Derived from Normal Neonatal Mice.” Eur. J. Immunol. 14, 435-441.

- G. Wennerstrom, L. Andrade and A. Coutinho. 1986. "The High Idiotypic Connectivity of "Natural" Newborn Antibodies is not Found in the Adult Mitogen-Reactive B Cell Repertoires." Eur. J. Immunol. 16, 82-87.

Irvine, D. H. and M. A. Savageau. 1985a. "Network Regulation of the Immune Response: Alternative Control Points for "Suppressor Modulation of Effector Lymphocytes." $J$. Immunol. 134, 2100-2116.

Kaufman, M., J. Urbain and R. Thomas. 1985. "Towards a Logical Analysis of the Immune Response." J. Theor, Biol. 114, 527-561.

Immune Response." J. Theor. Biol. 129, 141-162.

Jerne, N. K. 1974. "Towards a Network Theory of the Immune System." Ann. Immunol. (Inst. Pasteur) 125C, 373-389.

_ . 1984. "Idiotypic Networks and other Preconceived Ideas." Immunol. Rev. 79, 5-24. 
Levy, M. and A. Coutinho. 1987. "Long-Lived B Cells: Mitogen Activity as a Tool for Studying their Life-Spans." Eur. J. Immunol. 17, 295-298.

Möller, G. and G. Fernandez. 1986. "Functional Role of Autoantiidiotypic Antibodies in the Regulation of the Immune Response to Dextran." Ann. Immunol. (Inst. Pasteur) 137C, 94-96.

Novotny, J., M. Handschumacher and R. E. Bruccoleri. 1987. "Protein Antigenicity: A Static Surface Property." Immunol. Today 8, 26-31.

Perelson, A. S. 1988. "Towards a realistic model of the immune network". In Theoretical Immunology-Part Two. SFI Studies in the Science of Complexity, A. S. Perelson (Ed.), Vol. III, pp. 377-401. Reading, MA: Addison-Wesley.

Richter, P. H. 1975. “A Network Theory of the Immune System.” Eur. J. Immunol. 5, 350-354. 1978. "The Network Idea and the Immune Response." In Theoretical Immunology, G. I. Bell, A. S. Perelson and G. H. Pimbley (Eds), pp. 539-569. New York: Dekker.

Segel, L. A. and A. S. Perelson. 1988. "Computation in Shape Space: A New Approach to Immune Network Theory". In Theoretical Immunology-Part Two. SFI Studies in the Science of Complexity, A. S. Perelson (Ed.), Vol. III, pp. 321-343. Reading, MA: Addison-Wesley.

Seghers, M. 1979. "A Qualitative Study of an Idiotypic Cyclic Network." J. Theor. Biol. 80, 553-576.

Vakil, M. and J. F. Kearny. 1986. "Functional Characterization of Monoclonal Auto-AntiIdiotype Antibodies Isolated from the Early B Cell Repertoire of BALB/c Mice." Eur. $J$. Immunol. 16, 1151-1158.

Wikler, M., J-D. Franssen, C. Collignon, O. Leo, B. Mariamé, P. Van de Walle, D. De Groote and J. Urbain. 1979. "Idioptypic Regulation of the Immune System." J. Exp. Med. 150, 184-195.

Received 2 March 1988

Revised 1 July 1988 